\title{
A NONEXISTENCE RESULT FOR A NONLINEAR PDE WITH ROBIN CONDITION
}

\author{
BRAHIM KHODJA
}

Received 16 May 2005; Revised 24 April 2006; Accepted 4 May 2006

Under the assumption $\lambda>0$ and $f$ verifying $f(x, y, 0)=0$ in $D, 2 F(x, y, u)-u f(x, y, u) \geq$ $0, u \neq 0$, and if $\Omega=R \times D$, we show the convexity of function $E(t)=\iint_{D}|u(t, x, y)|^{2} d x d y$, where $u: \Omega \rightarrow \mathbb{R}$ is a solution of problem $\lambda\left(\partial^{2} u / \partial t^{2}\right)-(\partial / \partial x)(p(x, y)(\partial u / \partial x))-(\partial / \partial y)(q(x$, $y)(\partial u / \partial y))+f(x, y, u)=0$ in $\Omega, u+\varepsilon(\partial u / \partial n)=0$ on $\partial \Omega$, considered in $H^{2}(\Omega) \cap L^{\infty}(\Omega)$, $p, q: \bar{D} \rightarrow \mathbb{R}$ are two nonnull functions on $D, \varepsilon$ is a positive real number, and $D=] a_{1}, b_{1}$ [ $\times] a_{2}, b_{2}\left[,\left(F(x, y, s)=\int_{0}^{s} f(x, y, t) d t\right)\right.$.

Copyright (c) 2006 Hindawi Publishing Corporation. All rights reserved.

\section{Introduction}

In this paper we consider the question of absence of nontrivial solutions of the following boundary value problem:

$$
\begin{gathered}
\lambda \frac{\partial^{2} u}{\partial t^{2}}-\frac{\partial}{\partial x}\left(p(x, y) \frac{\partial u}{\partial x}\right)-\frac{\partial}{\partial y}\left(q(x, y) \frac{\partial u}{\partial y}\right)+f(x, y, u)=0 \quad \text { in } \Omega, \\
u+\varepsilon \frac{\partial u}{\partial n}=0 \quad \text { on } \partial \Omega,
\end{gathered}
$$

where

$$
p, q: \bar{D} \longrightarrow \mathbb{R}
$$

are two continuous functions $>0$ or $<0$ in $D, \varepsilon$ is a positive real number, and $f$ is a locally Lipschitz continuous function

$$
f: D \times \mathbb{R} \longrightarrow \mathbb{R}
$$


2 A nonexistence result for a nonlinear PDE

such that

$$
f(x, y, 0)=0 \quad \text { in } D \text {, }
$$

so that

$$
u \equiv 0
$$

is a solution of problem $(\mathrm{P})$.

This problem is considered in the Sobolev space

$$
H^{2}(\Omega) \cap L^{\infty}(\Omega),
$$

with

$$
\Omega=\mathbb{R} \times D, \quad D=] a_{1}, b_{1}[\times] a_{2}, b_{2}[.
$$

This question has interested much researchers and a significant number of works, were carried out. We quote by the way of examples the works of De Figueiredo and Jianfu [2], Esteban and Lions [3], Pucci and Serrin [6], Pohožaev [5], and Van der Vorst [7].

Esteban and Lions show that the Dirichlet problem

$$
\begin{gathered}
-\Delta u+f(u)=0, \quad u \in C^{2}(\bar{\Omega}), \\
u=0 \quad \text { on } \partial \Omega
\end{gathered}
$$

satisfying

$$
\begin{aligned}
& \nabla u \in L^{2}(\Omega), \quad f(0)=0, \\
& F(u)=\int_{0}^{u} f(s) d s \in L^{1}(\Omega),
\end{aligned}
$$

where $\Omega$ is a connected unbounded domain of $\mathbb{R}^{N}$ such that

$$
\exists \Lambda \in \mathbb{R}^{N}, \quad\|\Lambda\|=1, \quad\langle n(x), \Lambda\rangle \geq 0 \quad \text { on } \partial \Omega, \quad\langle n(x), \Lambda\rangle \neq 0,
$$

does not have nontrivial solution.

Berestycky et al. [1] established that the problem

$$
-\Delta u-u^{3}+u=0, \quad u \in H^{2}\left(\mathbb{R}^{2}\right),
$$


admits a radial solution. This solution satisfies

$$
\begin{array}{cc}
-\Delta u-u^{3}+u=0 & \text { in } \Omega=] 0,+\infty[\times \mathbb{R}, \\
\frac{\partial u}{\partial n}=0 & \text { on }\{0\} \times \mathbb{R} .
\end{array}
$$

This shows that the analog of the Dirichlet problem for the Neumann problem is not true. The problem

$$
\begin{gathered}
-\Delta u+u(u+1)(u+2)=0 \quad \text { in } \Omega=\mathbb{R} \times] 0, \alpha[, \\
\frac{\partial u}{\partial n}=0 \quad \text { on } \partial \Omega,
\end{gathered}
$$

considered in the Sobolev space

$$
H^{2}(\Omega) \cap L^{\infty}(\Omega)
$$

is still open in

$$
\Omega=\mathbb{R} \times] 0, a[(a<\pi) .
$$

These considerations motivated us to explore more this question of the absence of nontrivial solutions which presents a double interest. It makes it possible to solve an open problem, and to ensure then that the single solution is the null solution.

The aim of this work is to extend the results of [4] to problem (P). We prove in Section 2 a Pohožaev-type identity.

In Section 3, we combine Theorem 2.1 with other results to obtain for the semilinear elliptic problems a corollary of nonexistence of solutions. For the semilinear hyperbolic problems, we obtain in the same section an interesting result which shows that the KleinGordon align does not have nontrivial solutions.

Finally in Section 4, we give some examples to illustrate Theorems 3.1 and 3.5.

Let us denote by

$$
\Gamma=\partial \Omega=\mathbb{R} \times \partial D=\Gamma_{1} \cup \Gamma_{2} \cup \Gamma_{3} \cup \Gamma_{4},
$$

where

$$
\begin{aligned}
& \Gamma_{1}=\left\{\left(t, a_{1}, y\right), t \in \mathbb{R}, a_{2}<y<b_{2}\right\}, \\
& \Gamma_{2}=\left\{\left(t, b_{1}, y\right), t \in \mathbb{R}, a_{2}<y<b_{2}\right\}, \\
& \Gamma_{3}=\left\{\left(t, x, a_{2}\right), t \in \mathbb{R}, a_{1}<x<b_{1}\right\}, \\
& \Gamma_{4}=\left\{\left(t, x, b_{2}\right), t \in \mathbb{R}, a_{1}<x<b_{1}\right\},
\end{aligned}
$$

the boundary of $\Omega,(t, x, y)$, the generic point of $\Omega$,

$$
n\left(t, \xi_{1}, \xi_{2}\right)=\left(n_{1}\left(t, \xi_{1}, \xi_{2}\right), n_{2}\left(t, \xi_{1}, \xi_{2}\right), n_{3}\left(t, \xi_{1}, \xi_{2}\right)\right)
$$


the outward normal to $\partial \Omega$ at the point $\left(t, \xi_{1}, \xi_{2}\right), \partial^{2} u(t, x, y) / \partial s^{2}$, the second derivative of $u$ with respect to $s$ at point $(t, x, y)$, and

$$
\begin{aligned}
& \frac{\partial u}{\partial s \mid l_{1}}=\lim _{x \rightarrow a_{1}} \frac{\partial u}{\partial s}\left(t, x, b_{2}\right)=\lim _{y \rightarrow b_{2}} \frac{\partial u}{\partial s}\left(t, a_{1}, y\right), \\
& \frac{\partial u}{\partial s \mid l_{2}}=\lim _{x \rightarrow a_{1}} \frac{\partial u}{\partial s}\left(t, x, a_{2}\right)=\lim _{y \rightarrow a_{2}} \frac{\partial u}{\partial s}\left(t, a_{1}, y\right), \\
& \frac{\partial u}{\partial s \mid l_{3}}=\lim _{x \rightarrow b_{1}} \frac{\partial u}{\partial s}\left(t, x, b_{2}\right)=\lim _{y \rightarrow b_{2}} \frac{\partial u}{\partial s}\left(t, b_{1}, y\right), \\
& \frac{\partial u}{\partial s \mid l_{4}}=\lim _{x \rightarrow b_{1}} \frac{\partial u}{\partial s}\left(t, x, a_{2}\right)=\lim _{y \rightarrow a_{2}} \frac{\partial u}{\partial s}\left(t, b_{1}, y\right),
\end{aligned}
$$

where

$$
\begin{aligned}
& l_{1}=\Gamma_{1} \cap \Gamma_{4}=\left\{\left(t, a_{1}, b_{2}\right), t \in \mathbb{R}\right\}, \\
& l_{2}=\Gamma_{1} \cap \Gamma_{3}=\left\{\left(t, a_{1}, a_{2}\right), t \in \mathbb{R}\right\}, \\
& l_{3}=\Gamma_{2} \cap \Gamma_{4}=\left\{\left(t, b_{1}, b_{2}\right), t \in \mathbb{R}\right\}, \\
& l_{4}=\Gamma_{2} \cap \Gamma_{3}=\left\{\left(t, b_{1}, a_{2}\right), t \in \mathbb{R}\right\} .
\end{aligned}
$$

\section{Integral identity}

We now give an integral identity in the form of theorem.

Theorem 2.1. Let $u$ be an element of $H^{2}(\Omega) \cap L^{\infty}(\Omega)$, a solution of problem $(P)$, then for each $t \in \mathbb{R}$ and $\varepsilon>0$,

$$
\begin{aligned}
\iint_{D}\left[\frac{\lambda}{2}\left|\frac{\partial u}{\partial t}\right|^{2}+\frac{p(x, y)}{2}\left|\frac{\partial u}{\partial x}\right|^{2}+\frac{q(x, y)}{2}\left|\frac{\partial u}{\partial y}\right|^{2}+F(x, y, u)\right] d x d y \\
+\frac{1}{2 \varepsilon}\left(\int_{a_{1}}^{b_{1}}\left[p\left(x, a_{2}\right)\left|u\left(t, x, a_{2}\right)\right|^{2}+q\left(x, b_{2}\right)\left|u\left(t, x, b_{2}\right)\right|^{2}\right] d x\right. \\
\left.+\int_{a_{2}}^{b_{2}}\left[p\left(a_{1}, y\right)\left|u\left(t, a_{1}, y\right)\right|^{2}+q\left(b_{1}, y\right)\left|u\left(t, b_{1}, y\right)\right|^{2}\right] d y\right)=0 .
\end{aligned}
$$

Proof. For $t \in \mathbb{R}$, we consider a function $K$ defined by

$$
K(t)=\iint_{D}\left[\frac{\lambda}{2}\left|\frac{\partial u}{\partial t}\right|^{2}+\frac{p(x, y)}{2}\left|\frac{\partial u}{\partial x}\right|^{2}+\frac{q(x, y)}{2}\left|\frac{\partial u}{\partial y}\right|^{2}+F(x, y, u)\right] d x d y .
$$

The hypotheses on $u, p, q$, and $f$ imply that $K$ is absolutely continuous and thus differentiable almost everywhere on $\mathbb{R}$; we have

$$
K^{\prime}(t)=\iint_{D}\left[\lambda \frac{\partial u}{\partial t} \frac{\partial^{2} u}{\partial t^{2}}+p(x, y) \frac{\partial u}{\partial x} \frac{\partial^{2} u}{\partial x \partial t}+q(x, y) \frac{\partial u}{\partial y} \frac{\partial^{2} u}{\partial y \partial t}+\frac{\partial u}{\partial t} f(x, y, u)\right] d x d y .
$$


Fubini's theorem and an integration by part make it possible to write

$$
\begin{aligned}
\iint_{D} p(x, y)\left(\frac{\partial u}{\partial x} \frac{\partial^{2} u}{\partial x \partial t}\right)(t, x, y) d x d y \\
=\int_{a_{1}}^{b_{1}} \int_{a_{2}}^{b_{2}} p(x, y) \frac{\partial u}{\partial x} \frac{\partial^{2} u}{\partial x \partial t}(t, x, y) d x d y \\
=\int_{a_{2}}^{b_{2}}\left[\int_{a_{1}}^{b_{1}} p(x, y)\left(\frac{\partial u}{\partial x} \frac{\partial^{2} u}{\partial x \partial t}\right)(t, x, y) d x\right] d y \\
=\int_{a_{1}}^{b_{1}} \int_{a_{2}}^{b_{2}}-\frac{\partial}{\partial x}\left(p(x, y) \frac{\partial u}{\partial x}\right) \frac{\partial u}{\partial t}(t, x, y) d x d y \\
\quad+\int_{a_{2}}^{b_{2}}\left[p\left(b_{1}, y\right)\left(\frac{\partial u}{\partial x} \frac{\partial u}{\partial t}\right)\left(t, b_{1}, y\right)-p\left(a_{1}, y\right)\left(\frac{\partial u}{\partial x} \frac{\partial u}{\partial t}\right)\left(t, a_{1}, y\right)\right] d y
\end{aligned}
$$

In the same way, we find

$$
\begin{aligned}
\iint_{D} q(x, y) \frac{\partial u}{\partial y} \frac{\partial^{2} u}{\partial y \partial t}(t, x, y) d x d y \\
\quad=\int_{a_{1}}^{b_{1}} \int_{a_{2}}^{b_{2}}-\frac{\partial}{\partial y}\left(q(x, y) \frac{\partial u}{\partial y}\right) \frac{\partial u}{\partial t}(t, x, y) d x d y \\
\quad+\int_{a_{1}}^{b_{1}}\left[q\left(b_{1}, y\right)\left(\frac{\partial u}{\partial x} \frac{\partial u}{\partial t}\right)\left(t, b_{1}, y\right)-q\left(a_{1}, y\right)\left(\frac{\partial u}{\partial x} \frac{\partial u}{\partial t}\right)\left(t, a_{1}, y\right)\right] d x
\end{aligned}
$$

Replacing in (2.3), we find

$$
\begin{aligned}
K^{\prime}(t)= & \iint_{D}\left[\lambda \frac{\partial^{2} u}{\partial t^{2}}-\frac{\partial}{\partial x}\left(p(x, y) \frac{\partial u}{\partial x}\right)-\frac{\partial}{\partial y}\left(q(x, y) \frac{\partial u}{\partial y}\right)+f(x, y, u)\right] \frac{\partial u}{\partial t} d x d y \\
& +\int_{a_{1}}^{b_{1}}\left[q\left(x, b_{2}\right)\left(\frac{\partial u}{\partial x} \frac{\partial u}{\partial t}\right)\left(t, x, b_{2}\right)-q\left(x, a_{2}\right)\left(\frac{\partial u}{\partial x} \frac{\partial u}{\partial t}\right)\left(t, x, a_{2}\right)\right] d x \\
& +\int_{a_{2}}^{b_{2}}\left[p\left(b_{1}, y\right)\left(\frac{\partial u}{\partial x} \frac{\partial u}{\partial t}\right)\left(t, b_{1}, y\right)-p\left(a_{1}, y\right)\left(\frac{\partial u}{\partial x} \frac{\partial u}{\partial t}\right)\left(t, a_{1}, y\right)\right] d y .
\end{aligned}
$$

Let us write on $\partial \Omega$ the expression $u+\varepsilon \partial u / \partial n=0$ in an equivalent way:

$$
\begin{aligned}
u+\varepsilon \frac{\partial u}{\partial n}=0 \Longleftrightarrow u-\varepsilon \frac{\partial u}{\partial x}=0 & \text { on }\left\{\left(t, a_{1}, y\right), t \in \mathbb{R}, a_{2}<y<b_{2}\right\}, \\
u+\varepsilon \frac{\partial u}{\partial x}=0 & \text { on }\left\{\left(t, b_{1}, y\right), t \in \mathbb{R}, a_{2}<y<b_{2}\right\}, \\
u-\varepsilon \frac{\partial u}{\partial y}=0 & \text { on }\left\{\left(t, x, a_{2}\right), t \in \mathbb{R}, a_{1}<x<b_{1}\right\}, \\
u+\varepsilon \frac{\partial u}{\partial y}=0 & \text { on }\left(t, x, b_{2}\right), t \in \mathbb{R}, a_{1}<x<b_{1} .
\end{aligned}
$$


6 A nonexistence result for a nonlinear PDE

For $0<\varepsilon<+\infty$, we can write

$$
\begin{aligned}
\int_{a_{1}}^{b_{1}} & {\left[q\left(x, b_{2}\right)\left(\frac{\partial u}{\partial x} \frac{\partial u}{\partial t}\right)\left(t, x, b_{2}\right)-q\left(x, a_{2}\right) \frac{\partial u}{\partial x} \frac{\partial u}{\partial t}\left(t, x, a_{2}\right)\right] d x } \\
& =\frac{1}{\varepsilon}\left[\int_{a_{1}}^{b_{1}}\left[q\left(x, b_{2}\right)\left(u \frac{\partial u}{\partial t}\right)\left(t, x, b_{2}\right)-q\left(x, a_{2}\right)\left(u \frac{\partial u}{\partial t}\right)\left(t, x, a_{2}\right)\right] d x\right] \\
& =-\frac{1}{2 \varepsilon} \frac{d}{d t}\left(\int_{a_{1}}^{b_{1}}\left[q\left(x, b_{2}\right)\left|u\left(t, x, b_{2}\right)\right|^{2}+q\left(x, a_{2}\right)\left|u\left(t, x, a_{2}\right)\right|^{2}\right] d x\right)
\end{aligned}
$$

and in the same manner

$$
\begin{aligned}
\int_{a_{2}}^{b_{2}}\left[p\left(b_{1}, y\right)\left(\frac{\partial u}{\partial x} \frac{\partial u}{\partial t}\right)\left(t, b_{1}, y\right)-p\left(a_{1}, y\right)\left(\frac{\partial u}{\partial x} \frac{\partial u}{\partial t}\right)\left(t, a_{1}, y\right)\right] d y \\
=-\frac{1}{2 \varepsilon} \frac{d}{d t}\left(\int_{a_{2}}^{b_{2}}\left[p\left(b_{1}, y\right)\left|u\left(t, b_{1}, y\right)\right|^{2}+p\left(a_{1}, y\right)\left|u\left(t, a_{1}, y\right)\right|^{2}\right] d y\right)
\end{aligned}
$$

$K^{\prime}$, derivative of $K$, verifies therefore

$$
\begin{aligned}
\frac{d}{d t}\left(K(t)+\frac{1}{2 \varepsilon}(\right. & \int_{a_{1}}^{b_{1}}\left[q\left(x, b_{2}\right) u^{2}\left(t, x, b_{2}\right)+q\left(x, a_{2}\right) u^{2}\left(t, x, a_{2}\right)\right] d x \\
& \left.\left.+\int_{a_{2}}^{b_{2}}\left[p\left(b_{1}, y\right)\left|u\left(t, b_{1}, y\right)\right|^{2}+p\left(a_{1}, y\right)\left|u\left(t, a_{1}, y\right)\right|^{2}\right] d y\right)\right)=0 .
\end{aligned}
$$

Integrating with respect to $t$, we obtain

$$
\begin{aligned}
K(t)+\frac{1}{2 \varepsilon} & \left(\int_{a_{1}}^{b_{1}}\left[q\left(x, b_{2}\right) u^{2}\left(t, x, b_{2}\right)+q\left(x, a_{2}\right) u^{2}\left(t, x, a_{2}\right)\right] d x\right. \\
& \left.+\int_{a_{2}}^{b_{2}}\left[p\left(b_{1}, y\right)\left|u\left(t, b_{1}, y\right)\right|^{2}+p\left(a_{1}, y\right)\left|u\left(t, a_{1}, y\right)\right|^{2}\right] d y\right)=\text { const }
\end{aligned}
$$

and by noticing that

$$
\begin{aligned}
\int_{-\infty}^{+\infty}\left[K(t)+\frac{1}{2 \varepsilon}(\right. & \int_{a_{1}}^{b_{1}}\left[q\left(x, b_{2}\right) u^{2}\left(t, x, b_{2}\right)+q\left(x, a_{2}\right) u^{2}\left(t, x, a_{2}\right)\right] d x \\
& \left.\left.+\int_{a_{2}}^{b_{2}}\left[p\left(b_{1}, y\right)\left|u\left(t, b_{1}, y\right)\right|^{2}+p\left(a_{1}, y\right)\left|u\left(t, a_{1}, y\right)\right|^{2}\right] d y\right)\right] d t<\infty .
\end{aligned}
$$

The constant is null; this shows the theorem. 


\section{Main results}

The parameter $\lambda$ plays in fact an important part as it allows problem $(\mathrm{P})$ to be dealt with in two manners according to whether its value is positive or negative.

3.1. Semilinear hyperbolic problems. By employing identity (2.1) we obtain the following nonexistence result.

Theorem 3.1. Let, $\lambda>0$,

$$
p(x, y), q(x, y)>0 \quad \text { in } D
$$

and $f$ satisfying

$$
F(x, y, u) \geq 0, \quad u \neq 0
$$

Then problem $(P)$ considered in $H^{2}(\Omega) \cap L^{\infty}(\Omega)$ does not have nontrivial solutions.

Proof. Applying formula (2.1) we immediately obtain

$$
\frac{\partial u}{\partial t}(t, x, y)=\frac{\partial u}{\partial x}(t, x, y)=\frac{\partial u}{\partial y}(t, x, y)=0 \quad \text { in } \Omega
$$

The following theorem gives a nonexistence result if $f$ satisfies another type of nonlinearity.

Theorem 3.2. Let, $\lambda>0$,

$$
u \in H^{2}(\Omega) \cap L^{\infty}(\Omega)
$$

be a solution of problem $(P)$,

$$
p(x, y), q(x, y)>0 \text { or }<0 \quad \text { in } D \text {, }
$$

and $f$ a function verifying

$$
2 F(x, y, u)-u f(x, y, u) \geq 0, \quad u \neq 0 .
$$

Then the function

$$
E(t)=\iint_{D}|u(t, x, y)|^{2} d x d y
$$

is convex on $\mathbb{R}$.

Remark 3.3. The convexity of the function $E(t)$ on $\mathbb{R}$ implies the triviality of the solution $u(t, x, y)$ of the problem $(\mathrm{P})$. 
8 A nonexistence result for a nonlinear PDE

Proof. It is easy to see that almost everywhere in $\Omega$, we have

$$
\left(u \frac{\partial^{2} u}{\partial t^{2}}\right)(t, x, y)=\left(\frac{1}{2} \frac{\partial^{2}\left(u^{2}\right)}{\partial t^{2}}-\left|\frac{\partial u}{\partial t}\right|^{2}\right)(t, x, y)
$$

Let us multiply the first equation of $(\mathrm{P})$ by $u / 2$ and integrate the new align over $D$, we obtain

$$
\begin{aligned}
\iint_{D}[\lambda & \left.\frac{\partial^{2} u}{\partial t^{2}} \frac{u}{2}-\frac{\partial}{\partial x}\left(p(x, y) \frac{\partial u}{\partial x}\right) \frac{u}{2}-\frac{\partial}{\partial y}\left(q(x, y) \frac{\partial u}{\partial y}\right) \frac{u}{2}+\frac{1}{2} u f(x, y, u)\right](t, x, y) d x d y \\
= & \iint_{D}\left[\frac{\lambda}{4} \frac{\partial^{2}\left(u^{2}\right)}{\partial t^{2}}-\frac{\lambda}{2}\left|\frac{\partial u}{\partial t}\right|^{2}\right](t, x, y) d x d y \\
& +\iint_{D}\left[-\frac{\partial}{\partial x}\left(p(x, y) \frac{\partial u}{\partial x}\right) \frac{u}{2}-\frac{\partial}{\partial y}\left(q(x, y) \frac{\partial u}{\partial y}\right) \frac{u}{2}+\frac{1}{2} u f(x, y, u)\right](t, x, y) d x d y .
\end{aligned}
$$

Let us transform

$$
\begin{aligned}
\iint_{D}[ & \left.\frac{\partial}{\partial x}\left(p(x, y) \frac{\partial u}{\partial x}\right) \frac{u}{2}\right](t, x, y) d x d y \\
= & \int_{a_{1}}^{b_{1}} \int_{a_{2}}^{b_{2}} \frac{\partial}{\partial x}\left(p(x, y) \frac{\partial u}{\partial x}\right) \frac{u}{2}(t, x, y) d x d y \\
= & \int_{a_{2}}^{b_{2}}\left[\int_{a_{1}}^{b_{1}} \frac{\partial}{\partial x}\left(p(x, y) \frac{\partial u}{\partial x}\right) \frac{u}{2}(t, x, y) d x\right] d y \\
= & -\iint_{D} \frac{p(x, y)}{2}\left|\frac{\partial u(t, x, y)}{\partial x}\right|^{2} d x d y \\
& +\int_{a_{2}}^{b_{2}}\left[p\left(b_{1}, y\right)\left(\frac{u}{2} \frac{\partial u}{\partial x}\right)\left(t, b_{1}, y\right)-p\left(a_{1}, y\right)\left(\frac{u}{2} \frac{\partial u}{\partial x}\right)\left(t, a_{1}, y\right)\right] d y .
\end{aligned}
$$

Similary,

$$
\begin{aligned}
\iint_{D}\left[\frac{\partial}{\partial y}\left(q(x, y) \frac{\partial u}{\partial y}\right) \frac{u}{2}\right](t, x, y) d x d y \\
\quad=-\iint_{D} \frac{q(x, y)}{2}\left|\frac{\partial u(t, x, y)}{\partial y}\right|^{2} d x d y \\
\quad+\int_{a_{1}}^{b_{1}}\left[q\left(x, b_{2}\right)\left(\frac{u}{2} \frac{\partial u}{\partial y}\right)\left(t, x, b_{2}\right)-q\left(x, a_{2}\right)\left(\frac{u}{2} \frac{\partial u}{\partial y}\right)\left(t, x, a_{2}\right)\right] d x
\end{aligned}
$$


the substitution of these formulas in (3.7) gives

$$
\begin{gathered}
\iint_{D}\left[\frac{\lambda}{4} \frac{\partial^{2}\left(u^{2}\right)}{\partial t^{2}}-\frac{\lambda}{2}\left|\frac{\partial u}{\partial t}\right|^{2}-\frac{\partial}{\partial x}\left(p(x, y) \frac{\partial u}{\partial x}\right) \frac{u}{2}-\frac{\partial}{\partial y}\left(q(x, y) \frac{\partial u}{\partial y}\right) \frac{u}{2}+\frac{1}{2} u f(x, y, u)\right] d x d y \\
=-\frac{1}{2 \varepsilon}\left(\int_{a_{1}}^{b_{1}}\left[p\left(x, a_{2}\right)\left|u\left(t, x, a_{2}\right)\right|^{2}+p\left(x, b_{2}\right)\left|u\left(t, x, b_{2}\right)\right|^{2}\right] d x\right. \\
\left.+\int_{a_{2}}^{b_{2}}\left[q\left(a_{1}, y\right)\left|u\left(t, a_{1}, y\right)\right|^{2}+q\left(b_{1}, y\right)\left|u\left(t, b_{1}, y\right)\right|^{2}\right] d y\right),
\end{gathered}
$$

which combined with (2.1) yields

$$
\frac{\lambda}{4} \frac{d^{2}}{d t^{2}}\left(\iint_{D}|u(t, x, y)|^{2} d x d y\right)=\iint_{D}\left[\lambda\left|\frac{\partial u}{\partial t}\right|^{2}+F(x, y, u)-\frac{1}{2} u f(x, y, u)\right)(t, x, y] d x d y
$$

the assumption (B) and $\lambda>0$ enable us to affirm that

$$
\frac{\lambda}{4} \frac{d^{2}}{d t^{2}}\left(\iint_{D}|u(t, x, y)|^{2} d x d y\right) \geq \lambda \iint_{D}\left|\frac{\partial u(t, x, y)}{\partial t}\right|^{2} d x d y \geq 0
$$

This proves the theorem.

COROLlaRY 3.4. The result of Theorems 2.1 and 3.1 are still true if the condition of Robin is replaced either by Dirichlet or Neumann condition.

3.2. Semilinear elliptic problems. For the elliptic case, there is a nonexistence result which is stated as follows.

Theorem 3.5. Let

$$
u \in H^{2}(\Omega) \cap L^{\infty}(\Omega)
$$

be a solution of $(P), \lambda<0$, and $f$ satisfying

$$
2 F(x, y, u)-u f(x, y, u) \leq 0, \quad u \neq 0 .
$$

Then the function $E(t)$ defined in Theorem 3.2 is convex on $\mathbb{R}$.

Proof. The proof is similar to that of Theorem 3.2.

\section{Examples}

In this paragraph we will discuss some examples to demonstrate the use of Theorems 3.1 and 3.2. 
10 A nonexistence result for a nonlinear PDE

Example 4.1. Let

$$
\theta: D \longrightarrow R
$$

be a continuous function

$$
\begin{gathered}
p(x, y), q(x, y)>0 \quad \text { or } \quad<0 \text { in } D, \\
f(x, y, u)=\theta(x, y)|u|^{\gamma-1} u .
\end{gathered}
$$

Then problem

$$
\begin{gathered}
\lambda \frac{\partial^{2} u}{\partial t^{2}}-\frac{\partial}{\partial x}\left(p(x, y) \frac{\partial u}{\partial x}\right)-\frac{\partial}{\partial y}\left(q(x, y) \frac{\partial u}{\partial y}\right)+f(x, y, u)=0 \quad \text { in } \Omega, \\
u+\varepsilon \frac{\partial u}{\partial n}=0 \quad \text { on } \partial \Omega
\end{gathered}
$$

does not have nontrivial solutions. In the following situations:

$$
\begin{gathered}
\lambda>0, \\
p, q, \theta>0 \text { in } D,
\end{gathered}
$$

or

$$
\begin{aligned}
& \lambda>0, \\
& p(x, y), q(x, y)>0 \quad \text { or }<0, \quad \theta \leq 0 \text { in } D,
\end{aligned}
$$

we can see that in the first case

$$
F(x, y, u)=\frac{\theta(x, y)}{\gamma+1}|u|^{\gamma+1},
$$

and in the second case

$$
F(x, y, u)-\frac{1}{2} u f(x, y, u)=\theta(x, y)\left(\frac{1}{\gamma+1}-\frac{1}{2}\right)|u|^{\gamma+1} .
$$

Example 4.2. Let

$$
p, q, \rho: D \longrightarrow \mathbb{R}
$$

be continuous functions and

$$
f(x, y, u) \equiv u \text {. }
$$

The problem

$$
\begin{gathered}
\lambda \frac{\partial^{2} u}{\partial t^{2}}-\frac{\partial}{\partial x}\left(p(x, y) \frac{\partial u}{\partial x}\right)-\frac{\partial}{\partial y}\left(q(x, y) \frac{\partial u}{\partial y}\right)+\rho(x, y) u=0 \quad \text { in } \Omega, \\
u+\varepsilon \frac{\partial u}{\partial n}=0 \quad \text { on } \partial \Omega
\end{gathered}
$$

considered in $H^{2}(\Omega) \cap L^{\infty}(\Omega)$ does not have nontrivial solutions. 
Example 4.3. Let $\lambda>0$,

$$
\begin{gathered}
f(x, y, u)=m u-\theta_{1}(x, y)|u|^{r-1} u-\theta_{2}(x, y)|u|^{s-1} u \\
u \in H^{2}(\Omega) \cap L^{\infty}(\Omega)
\end{gathered}
$$

be a solution of

$$
\begin{gathered}
\lambda \frac{\partial^{2} u}{\partial t^{2}}-\frac{\partial}{\partial x}\left(p(x, y) \frac{\partial u}{\partial x}\right)-\frac{\partial}{\partial y}\left(q(x, y) \frac{\partial u}{\partial y}\right)+f(x, y, u)=0 \quad \text { in } \Omega \\
u+\varepsilon \frac{\partial u}{\partial n}=0 \quad \text { on } \partial \Omega
\end{gathered}
$$

where $m>0$ is the mass of a particle, let

$$
\theta_{1}, \theta_{2}: D \longrightarrow \mathbb{R}
$$

be two continuous nonnegative functions and $r$ and $s$ two numbers larger than one. The problem $(P)_{3}$ does not have nontrivial solutions. It suffices to remark that

$$
\begin{aligned}
& F(x, y, u)-\frac{1}{2} u f(x, y, u) \\
& \quad=\theta_{1}(x, y)\left(\frac{1}{2}-\frac{1}{r+1}\right)|u|^{r+1}+\theta_{2}(x, y)\left(\frac{1}{2}-\frac{1}{s+1}\right)|u|^{s+1} .
\end{aligned}
$$

Theorem 3.2 gives the desired result.

Example 4.4. Let

$$
\theta: D \longrightarrow \mathbb{R}
$$

be a nonnegative continuous function,

$$
p, q: D \longrightarrow \mathbb{R}
$$

two continuous functions $>0$ or $<0$ in $D$,

$$
f(x, y, u)=\theta(x, y)\left(\omega u+|u|^{\gamma-1} u\right),
$$

where $\omega$ is a real parameter and $\gamma \geq 1$; the problem

$$
\begin{gathered}
-\frac{\partial^{2} u}{\partial t^{2}}-\frac{\partial}{\partial x}\left(p(x, y) \frac{\partial u}{\partial x}\right)-\frac{\partial}{\partial y}\left(q(x, y) \frac{\partial u}{\partial y}\right)+f(x, y, u)=0 \quad \text { in } \Omega \\
u+\varepsilon \frac{\partial u}{\partial n}=0 \quad \text { on } \partial \Omega
\end{gathered}
$$

does not have nontrivial solutions in $H^{2}(\Omega) \cap L^{\infty}(\Omega)$. 
12 A nonexistence result for a nonlinear PDE

\section{Acknowledgment}

The author would like to thank the referee for several helpful comments.

\section{References}

[1] H. Berestycky, T. Gallouet, and O. Kavian, Equations de champs scalaires non-linéaires dans le plan, Comptes Rendus des Séances de l'Académie des Sciences. Série I. Mathématique 297 (1983), 307-310.

[2] D. G. De Figueiredo and Y. Jianfu, Decay, symmetry and existence of solutions of semilinear elliptic systems, Nonlinear Analysis 33 (1998), no. 3, 211-234.

[3] M. J. Esteban and P.-L. Lions, Existence and nonexistence results for semilinear elliptic problems in unbounded domains, Proceedings of the Royal Society of Edinburgh. Section A 93 (1982/1983), no. 1-2, 1-14.

[4] B. Khodja, Nonexistence of solutions for semilinear equations and systems in cylindrical domains, Communications on Applied Nonlinear Analysis 7 (2000), no. 4, 19-30.

[5] S. I. Pohožaev, Eigenfunctions of the equation $\Delta u+\lambda f(u)=0$, Soviet Mathematics. Doklady 6 (1965), 1408-1411.

[6] P. Pucci and J. Serrin, A general variational identity, Indiana University Mathematics Journal 35 (1986), no. 3, 681-703.

[7] R. C. A. M. Van der Vorst, Variational identities and applications to differential systems, Archive for Rational Mechanics and Analysis 116 (1992), no. 4, 375-398.

Brahim Khodja: Department of Mathematics, Faculty of Sciences, University of Annaba, P.O. Box 12, Annaba, Algeria

E-mail address: bmkhodja@yahoo.fr 


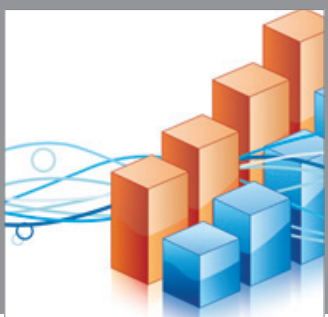

Advances in

Operations Research

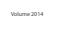

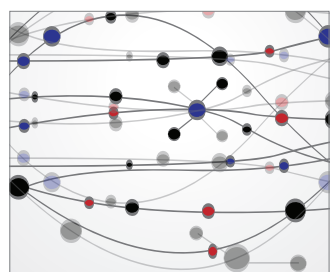

\section{The Scientific} World Journal
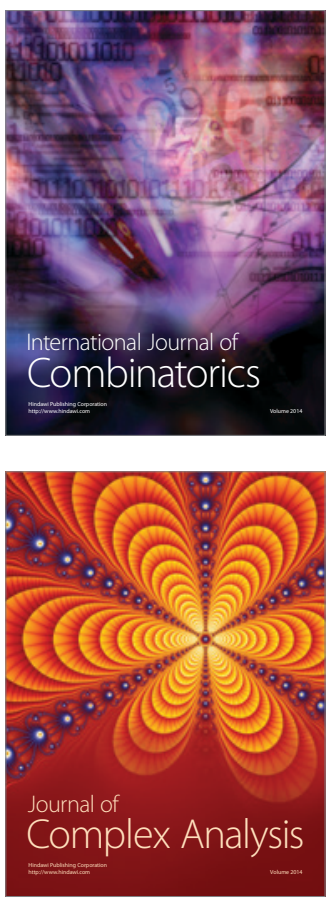

International Journal of

Mathematics and

Mathematical

Sciences
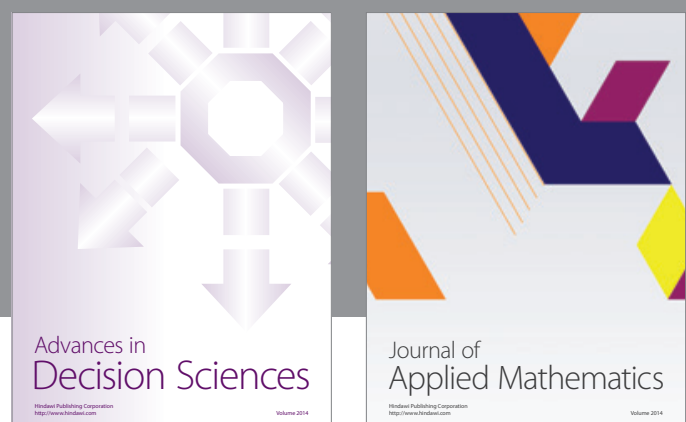

Journal of

Applied Mathematics
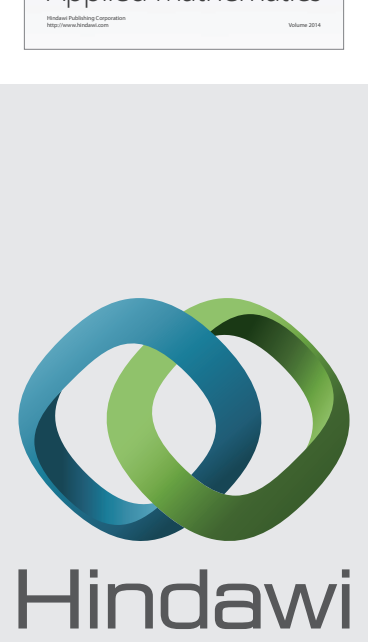

Submit your manuscripts at http://www.hindawi.com
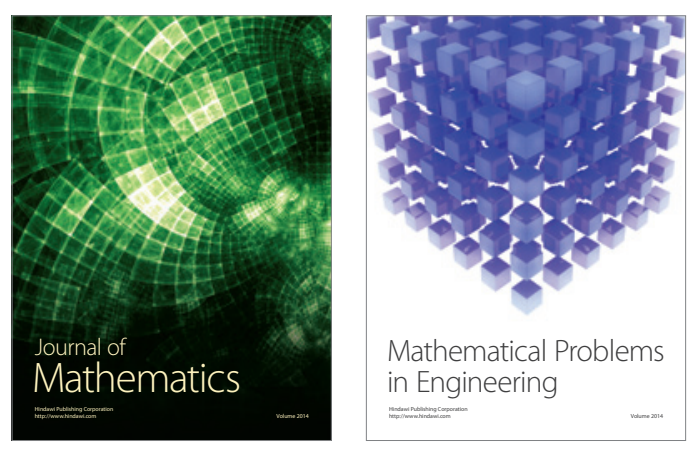

Mathematical Problems in Engineering
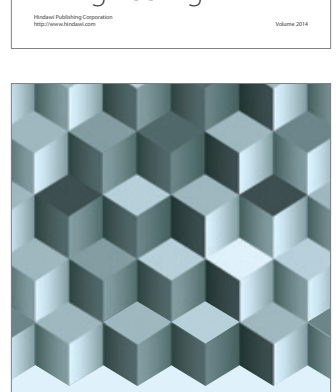

Journal of

Function Spaces
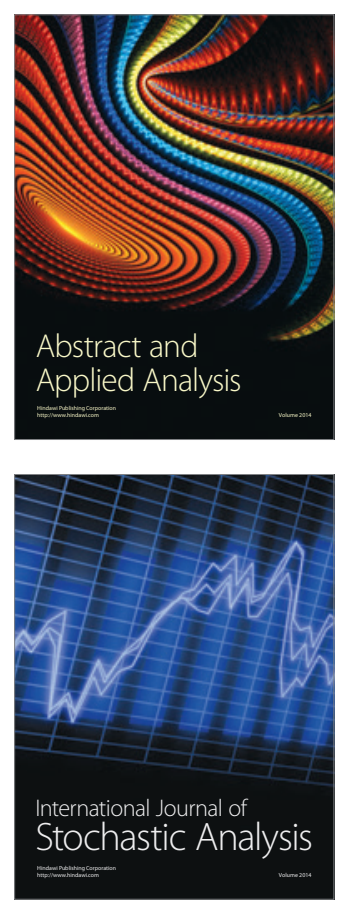

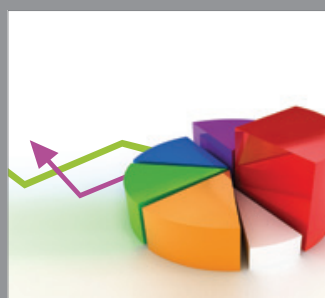

ournal of

Probability and Statistics

Promensencen
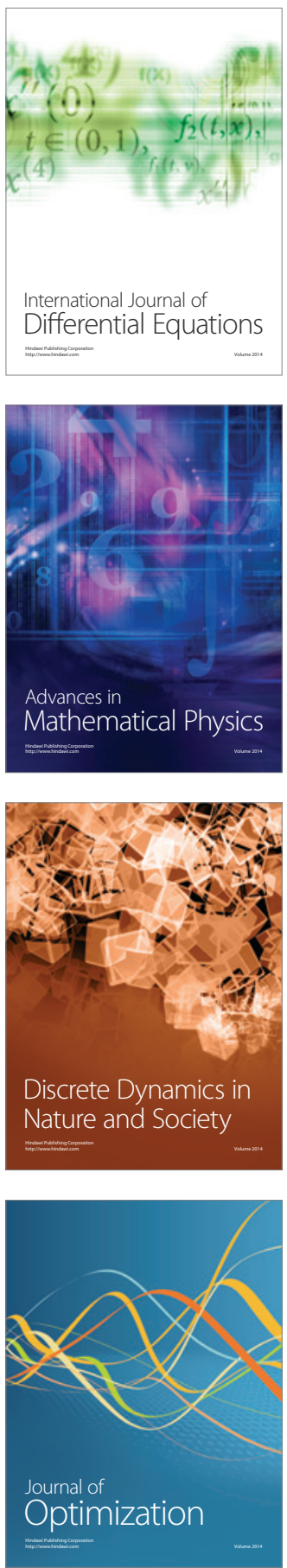